\title{
MUTUAL EXISTENCE OF PRODUCT INTEGRALS
}

\author{
JON C. HELTON
}

\begin{abstract}
Definitions and integrals are of the subdivisionrefinement type, and functions are from $R \times R$ to $R$, where $R$ represents the real numbers. Let $O M^{\circ}$ be the class of functions $G$ such that $\prod_{x} \prod^{r}(1+G)$ exists for $a \leqq x<y \leqq b$ and $\int_{a}^{b}|1+G-\Pi(1+G)|=0$. Let $O P^{\circ}$ be the class of functions $G$ such that $\left|\prod_{q=i}^{j}\left(1+G_{q}\right)\right|$ is bounded for refinements $\left\{x_{a}\right\}_{a=0}^{n}$ of a suitable subdivision of $[a, b]$. If $F$ and $G$ are functions from $R \times R$ to $R$ such that $F \in O P^{\circ}$ on $[a, b]$, $\lim _{x, y \rightarrow p^{+}} F(x, y)$ and $\lim _{x, y \rightarrow p^{-}} F(x, y)$ exist and are zero for $p \in$ $[a, b]$, each of $\lim _{x \rightarrow p^{+}} F(p, x), \lim _{x \rightarrow p^{-}} F(x, p), \lim _{x \rightarrow p^{+}} G(p, x)$ and $\lim _{x \rightarrow p^{-}} G(x, p)$ exist for $p \in[a, b]$, and $G$ has bounded variation on $[a, b]$, then any two of the following statements imply the other: (1) $F+G \in O M^{\circ}$ on $[a, b]$, (2) $F \in O M^{\circ}$ on $[a, b]$, and (3) $G \in O M^{\circ}$ on $[a, b]$.
\end{abstract}

All integrals and definitions are of the subdivision-refinement type, and functions are from $R \times R$ to $R$, where $R$ represents the set of real numbers. Furthermore, functions are assumed to be defined only for elements $\{x, y\}$ of $R \times R$ such that $x<y$. If $D=\left\{x_{a}\right\}_{q=0}^{n}$ is a subdivision of $[a, b]$, then $D(I)=\left\{\left[x_{q-1}, x_{q}\right]\right\}_{q=1}^{n}$ and $G_{q}=G\left(x_{q-1}, x_{q}\right)$. Further, $\left\{x_{q r}\right\}_{r=0}^{n(a)}$ represents a subdivision of the interval $\left[x_{a-1}, x_{q}\right]$ and $G_{a r}=G\left(x_{a, r-1}, x_{a r}\right)$. The statement that $\int_{a}^{b} G$ exists means there exists a number $L$ such that, if $\varepsilon>0$, then there exists a subdivision $D$ of $[a, b]$ such that if $J$ is a refinement of $D$, then

$$
\left|L-\sum_{J(I)} G\right|<\varepsilon .
$$

The statement that ${ }_{a} \Pi^{b}(1+G)$ exists means there exists a number $L$ such that, if $\varepsilon>0$, then there exists a subdivision $D$ of $[a, b]$ such that if $J$ is a refinement of $D$, then

$$
\left|L-\prod_{J(I)}(1+G)\right|<\varepsilon .
$$

Further, $G \in O A^{\circ}$ on $[a, b]$ only if $\int_{a}^{b} G$ exists and $\int_{a}^{b}\left|G-\int G\right|=0$, and

Presented to the Society, October 30, 1972; received by the editors October 6, 1972. AMS (MOS) subject classifications (1970). Primary 26A39, 26A42.

Key words and phrases. Product integral, sum integral, subdivision-refinement integral, interval function.

(c) American Mathematical Society 1974 
$G \in O M^{\circ}$ on $[a, b]$ only if ${ }_{x} \Pi^{y}(1+G)$ exists for $a \leqq x<y \leqq b$ and $\int_{a}^{b}\left|1+G-\prod(1+G)\right|=0$.

The statements that $G$ is bounded, $G \in O P^{\circ}, G \in O Q^{\circ}$ and $G \in O B^{\circ}$ on $[a, b]$ mean there exist a subdivision $D$ of $[a, b]$ and positive numbers $B$ and $\beta$ such that if $J=\left\{x_{a}\right\}_{a=0}^{n}$ is a refinement of $D$, then

(1) $|G(u)|<B$ for $u \in J(I)$,

(2) $\left|\prod_{q=r}^{s}\left(1+G_{q}\right)\right|<B$ for $1 \leqq r \leqq s \leqq n$,

(3) $\left|\prod_{q=r}^{s}\left(1+G_{q}\right)\right|>\beta$ for $1 \leqq r \leqq s \leqq n$, and

(4) $\sum_{J(I)}|G|<B$,

respectively.

If $G$ is a function, then $G \in S_{1}$ on $[a, b]$ only if $\lim _{x, y \rightarrow p^{+}} G(x, y)$ and $\lim _{x, y \rightarrow p^{-}} G(x, y)$ exist and are zero for $p \in[a, b]$, and $G \in S_{2}$ on $[a, b]$ only if $\lim _{x \rightarrow p^{+}} G(p, x)$ and $\lim _{x \rightarrow p^{-}} G(x, p)$ exist for $p \in[a, b]$. Further, $G \in O L^{\circ}$ on $[a, b]$ only if $\lim _{x, y \rightarrow p^{+}} G(x, y), \lim _{x, y \rightarrow p^{-}} G(x, y), \lim _{x \rightarrow p^{+}} G(p, x)$ and $\lim _{x \rightarrow p^{-}} G(x, p)$ exist for $p \in[a, b]$. See B. W. Helton [2] and J. S. MacNerney [7] for additional details.

LEMMA 1.1. If $F$ and $G$ are functions from $R \times R$ to $R$ such that $F \in O P^{\circ}$ on $[a, b]$ and $G \in O B^{\circ}$ on $[a, b]$, then $F+G \in O P^{\circ}$ on $[a, b]$.

Lemma 1.1 is part of a previous result by the author [5, Theorem 1].

LEMMA 1.2. If $G$ is a function from $R \times R$ to $R$ such that $\int_{a}^{b} G$ exists, then $G \in O A^{\circ}$ on $[a, b]$.

Lemma 1.2 is due to A. Kolmogoroff [6, p. 669]. The reader is also referred to results by W. D. L. Appling [1, Theorems 1, 2, p. 155] and B. W. Helton [2, Theorem 4.1, p. 304].

LEMMA 1.3. If $G$ is a function from $R \times R$ to $R$ such that $G \in O B^{\circ}$ on $[a, b]$, then the following statements are equivalent:

(1) $G \in O M^{\circ}$ on $[a, b]$,

(2) $G \in O A^{\circ}$ on $[a, b]$, and

(3) $\int_{a}^{b} G$ exists.

B. W. Helton [2, Theorem 3.4 , p. 301] shows that (1) and (2) are equivalent. Further, by Lemma 1.2, (2) and (3) are equivalent.

LEMMA 1.4. If $F$ and $G$ are functions from $R \times R$ to $R$ such that $F \in O M^{\circ}$, $O P^{\circ}$ and $S_{1} \cap S_{2}$ on $[a, b]$ and $G \in O M^{\circ}$ and $O B^{\circ}$ on $[a, b]$, then $F+G \in O M^{\circ}$ on $[a, b]$.

Lemma 1.4 is proved in a previous paper by the author [5, Theorem 2]. In the original version [5, Theorem 2] the theorem is stated with the requirement that $\int_{a}^{b} G$ exist rather than $G \in O M^{\circ}$ on $[a, b]$. However, Lemma 1.3 establishes the equivalence of the two forms. 
LEMMA 1.5. If $E$ is a finite set of points from $[a, b]$ and $F, G$ and $H$ are functions from $R \times R$ to $R$ such that

(1) $G \in O B^{\circ}$ and $S_{2}$ on $[a, b]$,

(2) $H \in O P^{\circ}$ and $S_{1} \cap S_{2}$ on $[a, b]$,

(3) $H+G \in O M^{\circ}$ on $[a, b]$, and

(4) $F \in S_{2}$ on $[a, b]$ and if $a \leqq x<y \leqq b$, then $F(x, y)=H(x, y)$ if $x \notin E$ and $y \notin E$,

then $F+G \in O M^{\circ}$ on $[a, b]$.

Proof. Lemma 1.1 establishes that $H+G \in O P^{\circ}$ on $[a, b]$. Further, $H+G \in S_{1} \cap S_{2}$ on $[a, b]$. Let $H^{\prime}$ be the function defined on $[a, b]$ such that if $a \leqq x<y \leqq b$, then

(1) $H^{\prime}(x, y)=0$ if $x \notin E$ and $y \notin E$, and

(2) $H^{\prime}(x, y)=F(x, y)-H(x, y)$ if $x \in E$ or $y \in E$.

Thus, $H^{\prime} \in O M^{\circ}$ and $O B^{\circ}$ on $[a, b]$. Therefore, by Lemma 1.4, $H+G+H^{\prime}$ is in $O M^{\circ}$ on $[a, b]$. Hence, since $H+G+H^{\prime} \equiv F+G$ on $[a, b], F+G \in$ $O M^{\circ}$ on $[a, b]$.

LEMMA 1.6. If $G$ is a bounded function from $R \times R$ to $R$ such that $\prod_{a}^{b}(1+G)$ exists and is not zero, then $G \in O P^{\circ}$ and $O Q^{\circ}$ on $[a, b]$.

Lemma 1.6 is a special case of a previous result by the author [4, Theorem 2].

LEMMA 1.7. If $G$ is a bounded function from $R \times R$ to $R$ such that $G \in$ $O M^{\circ}$ on $[a, b]$ and $1+G$ is bounded away from zero on $[a, b]$, then $G \in O P^{\circ}$ and $O Q^{\circ}$ on $[a, b]$.

Proof. Since $G \in O M^{\circ}$ on $[a, b]$ and $1+G$ is bounded away from zero on $[a, b], \Pi^{b}(1+G)$ exists and is not zero. Therefore, it follows from Lemma 1.6 that $G \in O P^{\circ}$ and $O Q^{\circ}$ on $[a, b]$.

LEMMA 1.8. If $G$ is a function from $R \times R$ to $R$ such that $G \in O B^{\circ}$ on $[a, b]$ and $1+G$ is bounded away from zero on $[a, b]$, then $G \in O Q^{\circ}$ on $[a, b]$.

Lemma 1.8 is a special case of a previous result by the author [5, Theorem 3].

LEMMA 1.9. If $G$ is a bounded function from $R \times R$ to $R$ such that ${ }_{a} \Pi^{b}(1+G)$ exists and is not zero, then $G \in O M^{\circ}$ on $[a, b]$.

Lemma 1.9 follows from Lemma 1.6 and a result of B. W. Helton [2, Theorem 4.2, p. 305].

LEMMA 1.10. If $G$ is a function from $R \times R$ to $R$ such that $G \in O B^{\circ}$ and $S_{2}$ on $[a, b]$ and ${ }_{x} \Pi^{y}(1+G)$ exists for $a \leqq x<y \leqq b$, then $G \in O M^{\circ}$ on $[a, b]$. 
ProOf. Let $\varepsilon>0$. Since $G \in O B^{\circ}$ on $[a, b]$, there exist a subdivision $D_{0}$ of $[a, b]$ and a number $B>1$ such that if $\left\{x_{i}\right\}_{i=0}^{n}$ is a refinement of $D_{0}$ and $1 \leqq r \leqq s \leqq n$, then

$$
\left|\prod_{i=r}^{s}\left(1+G_{i}\right)\right|<B
$$

There exists a subdivision $E=\left\{w_{a}\right\}_{q=0}^{t}$ of $[a, b]$ such that if $1 \leqq q \leqq t$ and $w_{a-1}<x<y<w_{a}$, then $|G(x, y)|<\frac{1}{2}$. Further, there exist sequences $\left\{u_{a}\right\}_{a=1}^{t}$ and $\left\{v_{a}\right\}_{q=1}^{t}$ such that

(1) $w_{a-1}<u_{q}<v_{a}<w_{q}$,

(2) if $w_{a-1}<x<y \leqq u_{a}$, then

$$
\left|G\left(w_{q-1}, x\right)-G\left(w_{q-1}, y\right)\right|<\varepsilon(8 t)^{-1},
$$

(3) if $w_{q-1}<x<u_{q}$ and $J$ is a subdivision of $\left[x, u_{q}\right]$, then

$$
\sum_{J(I)}|G|<\varepsilon\left(8 B^{3} t\right)^{-1}
$$

(4) if $u_{a} \leqq x<y<w_{a}$, then

$$
\left|G\left(x, w_{q}\right)-G\left(y, w_{q}\right)\right|<\varepsilon(8 t)^{-1},
$$

and

(5) if $v_{q}<x<w_{q}$ and $J$ is a subdivision of $\left[v_{q}, x\right]$, then

$$
\sum_{J(I)}|G|<\varepsilon\left(8 B^{3} t\right)^{-1}
$$

We know from the hypothesis that ${ }_{u_{q}} \prod^{v_{a}}(1+G)$ exists for $1 \leqq q \leqq t$. Further, it follows from Lemma 1.8 that each of these integrals is nonzero. Thus, Lemma 1.9 implies that $G \in O M^{\circ}$ on $\left[u_{q}, v_{q}\right]$. Hence, for $1 \leqq q \leqq t$, there exists a subdivision $D_{a}$ of $\left[u_{a}, v_{a}\right]$ such that if $J=\left\{x_{i}\right\}_{i=0}^{n}$ is a refinement of $D_{q}$ and $\left\{x_{i j}\right\}_{j=0}^{n(i)}$ is a subdivision of $\left[x_{i-1}, x_{i}\right]$, then

$$
\sum_{i=1}^{n}\left|1+G_{i}-\prod_{j=1}^{n(i)}\left(1+G_{i j}\right)\right|<\varepsilon(8 t)^{-1} .
$$

Let $D$ denote the subdivision $\bigcup_{a=0}^{t} D_{q} \cup E$ of $[a, b]$. Suppose $\left\{x_{i}\right\}_{i=0}^{n}$ is a refinement of $D$. Let $\left\{x_{w(i)}\right\}_{i=0}^{t}$ be the subsequence of $\left\{x_{i}\right\}_{i=0}^{n}$ such that $x_{w(i)}=w_{i}$. Further, let $\left\{x_{u(i)}\right\}_{i=1}^{t}$ and $\left\{x_{v(i)}\right\}_{i=1}^{t}$ be the subsequences of $\left\{x_{i}\right\}_{i=0}^{n}$ such that $x_{u(i)}=u_{i}$ and $x_{v(i)}=v_{i}$. Let $T(q), U(q)$ and $V(q)$ denote $\{i\}_{i=u(q)+1}^{v(q)},\{i\}_{i=w(q-1)+1}^{u(q)}$ and $\{i\}_{i=v(q)+1}^{w(q)}$, respectively. Further, let $U, V, U^{\prime}(q)$ and $V^{\prime}(q)$ denote $\{w(i)+1\}_{i=0}^{t-1},\{w(i)\}_{i=1}^{t},\{i\}_{i=v(q-1)+2}^{u(q)}$ and $\{i\}_{i=v(q)+1}^{w(q)-1}$, respectively. Finally, let $S(q)$ and $S^{\prime}(q)$ represent $U(q) \cup V(q)$ and $U^{\prime}(q) \cup$ $V^{\prime}(q)$, respectively. 
For $1 \leqq i \leqq n$, there exists a subdivision $\left\{x_{i j}\right\}_{j=0}^{n(i)}$ of $\left[x_{i-1}, x_{i}\right]$ such that

$$
\left|\prod_{j=1}^{n(i)}\left(1+G_{i j}\right)-{ }_{x_{i-1}} \prod^{x_{i}}(1+G)\right|<\varepsilon(8 n)^{-1} \text {. }
$$

Let $H_{i}$ represent $1+G_{i}-\prod_{j=1}^{n(i)}\left(1+G_{i j}\right)$. Thus,

$$
\begin{aligned}
& \sum_{i=1}^{n}\left|1+G_{i}-{ }_{x_{i-1}} \prod^{x_{i}}(1+G)\right|<\sum_{i=1}^{n}\left|H_{i}\right|+\left[\varepsilon(8 n)^{-1}\right] n \\
& =\sum_{q=1}^{t} \sum_{i \in S(q)}\left|H_{i}\right|+\sum_{q=1}^{t} \sum_{i \in T(q)}\left|H_{i}\right|+\frac{\varepsilon}{8} \\
& <\sum_{q=1}^{t} \sum_{i \in S(q)}\left|H_{i}\right|+\left[\varepsilon(8 t)^{-1}\right] t+\frac{\varepsilon}{8} \\
& =\sum_{i \in U \cup V}\left|H_{i}\right|+\sum_{q=1}^{t} \sum_{i \in S^{\prime}(\alpha)}\left|H_{i}\right|+\frac{\varepsilon}{4} \\
& =\sum_{i \in U \cup V}\left|H_{i}\right| \\
& +\sum_{q=1}^{t} \sum_{i \in S^{\prime}(q)} \mid\left\{1+G_{i}\right\}-\left\{1+\sum_{j=1}^{n(i)}\left[\prod_{r=1}^{j-1}\left(1+G_{i r}\right)\right]\right. \\
& \left.\cdot\left[G_{i j}\right]\left[\prod_{s=j+1}^{n(i)}\left(1+G_{i s}\right)\right]\right\} \mid+\frac{\varepsilon}{4} \\
& \leqq \sum_{i \in U \cup V}\left|H_{i}\right|+\sum_{q=1}^{t} \sum_{i \in S^{\prime}(q)}\left[\left|G_{i}\right|+B^{2} \sum_{j=1}^{n(i)}\left|G_{i j}\right|\right]+\frac{\varepsilon}{4} \\
& <\sum_{i \in U \cup V}\left|H_{i}\right|+\left[\varepsilon\left(8 B^{3} t\right)^{-1}\right] t+B^{2}\left[\varepsilon\left(8 B^{3} t\right)^{-1}\right] t+\frac{\varepsilon}{4} \\
& <\sum_{i \in U \cup V}\left|H_{i}\right|+\frac{\varepsilon}{2} \\
& \leqq ! \sum_{i \in U}\left|\left(1+G_{i}\right)-\left(1+G_{i 1}\right)\right|+\sum_{i \in U}\left|1+G_{i 1}\right|\left|-1+\prod_{j=2}^{n(i)}\left(1+G_{i j}\right)\right| \\
& +\sum_{i \in V}\left|\left(1+G_{i}\right)-\left(1+G_{i, n(i)}\right)\right| \\
& +\sum_{i \in V}\left|1+G_{i, n(i)}\right|\left|-1+\prod_{j=1}^{n(i)-1}\left(1+G_{i j}\right)\right|+\frac{\varepsilon}{2} \\
& \leqq \sum_{i \in U}\left|G_{i}-G_{i 1}\right|+B \sum_{i \in U} \mid-1+\left\{1+\sum_{j=2}^{n(i)}\left[\prod_{r=2}^{j-1}\left(1+G_{i r}\right)\right]\left[G_{i j}\right]\right. \\
& \left.\cdot\left[\prod_{s=j+1}^{n(i)}\left(1+G_{i s}\right)\right]\right\} \mid
\end{aligned}
$$




$$
\begin{aligned}
& +\sum_{i \in V}\left|G_{i}-G_{i, n(i)}\right|+B \sum_{i \in V} \mid-1+\left\{1+\sum_{j=1}^{n(i)-1}\left[\prod_{r=1}^{j-1}\left(1+G_{i r}\right)\right]\left[G_{i j}\right]\right. \\
& \left.<\left[\varepsilon\left(\prod_{s=j+1}^{n(i)-1}\left(1+G_{i s}\right)\right]\right\}\right]+\frac{\varepsilon}{2} \\
& \quad+\left[\varepsilon(8 t)^{-1}\right] t+B^{3} \sum_{i \in V} \sum_{j=2}^{n(i)}\left|G_{i j}\right| \\
& <\sum_{j=1}^{n(i)-1}\left|G_{i j}\right|+\frac{\varepsilon}{2} \\
& =\varepsilon .
\end{aligned}
$$

Therefore, $G \in O M^{\circ}$ on $[a, b]$.

Lemma 1.10 is not true if only ${ }_{a} \Pi^{b}(1+G)$ is required to exist rather than ${ }_{x} \Pi^{y}(1+G)$ for $a \leqq x<y \leqq b$. For example, consider the function $G$ defined on $[0,1]$ such that, for $0 \leqq x<y \leqq 1$,

(1) $G(0, x)=-1$,

(2) $G(x, y)=y-x$ if $x \neq 0$ and $y$ is irrational, and

(3) $G(x, y)=x-y$ if $x \neq 0$ and $y$ is rational.

Thus, $G \in O B^{\circ}$ and $S_{2}$ on $[a, b]$ and $a \Pi^{b}(1+G)$ exists and is zero. However, ${ }_{x} \Pi^{v}(1+G)$ does not exist for $a \leqq x<y \leqq b$, and thus, $G \notin O M^{\circ}$ on $[a, b]$.

LEMMA 1.11. If $H$ and $G$ are functions from $R \times R$ to $R$ such that $H \in O L^{\circ}$ on $[a, b], G \in O B^{\circ}$ on $[a, b]$ and either $G \in O M^{\circ}$ on $[a, b]$ or $G \in O A^{\circ}$ on $[a, b]$, then $H G \in O M^{\circ}$ and $O A^{\circ}$ on $[a, b]$.

Lemma 1.11 is a modification of a result of B. W. Helton [3, Theorem 2, p. 494] obtained by using Lemma 1.3.

THEOREM 1. If $F$ and $G$ are functions from $R \times R$ to $R$ such that $F \in O P^{\circ}$ and $S_{1} \cap S_{2}$ on $[a, b]$ and $G \in O B^{\circ}$ and $S_{2}$ on $[a, b]$, then any two of the following statements imply the other:

(1) $F+G \in O M^{\circ}$ on $[a, b]$,

(2) $F \in O M^{\circ}$ on $[a, b]$, and

(3) $G \in O M^{\circ}$ on $[a, b]$.

ProOf $(1,2 \rightarrow 3)$. There exists a subdivision $E=\left\{w_{i}\right\}_{i=0}^{t}$ of $[a, b]$ such that if $1 \leqq i \leqq t$ and $w_{i-1}<x<y<w_{i}$, then $|F(x, y)|<\frac{1}{2}$. Let $F^{\prime}(x, y)=$ $F(x, y)$ if $x \notin E$ and $y \notin E$, and let $F^{\prime}(x, y)=0$ if $x \in E$ or $y \in E$. Thus, $\left(1+F^{\prime}\right)^{-1}$ is in $O L^{\circ}$ on $[a, b]$. Further, it follows from Lemma 1.5 that $F^{\prime}+G \in O M^{\circ}$ on $[a, b]$ and $F^{\prime} \in O M^{\circ}$ on $[a, b]$. Hence, since $F^{\prime} \in O M^{\circ}$ on 
$[a, b]$, Lemma 1.7 implies that $F^{\prime} \in O Q^{\circ}$ on $[a, b]$. Also, note that $G\left(1+F^{\prime}\right)^{-1}$ is in $O B^{\circ}$ and $O P^{\circ}$ on $[a, b]$.

We now establish that ${ }_{x} \Pi^{y}\left[1+G\left(1+F^{\prime}\right)^{-1}\right]$ exists by using the Cauchy criterion for product integrals, where $a \leqq x<y \leqq b$. Let $\varepsilon>0$. There exist a subdivision $D$ of $[x, y]$ and positive numbers $B$ and $\beta$ such that if $J$ and $K$ are refinements of $D$, then

(1) $\left|\prod_{J(I)}\left(1+F^{\prime}\right)\right|>\beta$,

(2) $\left|\prod_{J(I)}\left[1+G\left(1+F^{\prime}\right)^{-1}\right]\right|<B$,

(3) $\left|\prod_{J(I)}\left(1+F^{\prime}\right)-\prod_{K^{\prime}(I)}\left(1+F^{\prime}\right)\right|<\beta \varepsilon(2 B)^{-1}$, and

(4) $\left|\prod_{J(I)}\left(1+F^{\prime}+G\right)-\prod_{K(I)}\left(1+F^{\prime}+G\right)\right|<\beta \varepsilon / 2$.

Suppose $J$ and $K$ are refinements of $D$. Thus,

$$
\begin{aligned}
\frac{\beta \varepsilon}{2}> & \left|\prod_{J(I)}\left(1+F^{\prime}+G\right)-\prod_{K^{\prime}(I)}\left(1+F^{\prime}+G\right)\right| \\
= & \mid\left\{\prod_{J(I)}\left(1+F^{\prime}\right)\right\}\left\{\prod_{J(I)}\left[1+G\left(1+F^{\prime}\right)^{-1}\right]\right\} \\
& -\left\{\prod_{K^{\prime}(I)}\left(1+F^{\prime}\right)\right\}\left\{\prod_{K^{\prime}(I)}\left[1+G\left(1+F^{\prime}\right)^{-1}\right]\right\} \mid \\
\geqq & \left|\prod_{J(I)}\left(1+F^{\prime}\right)\right|\left|\prod_{J(I)}\left[1+G\left(1+F^{\prime}\right)^{-1}\right]-\prod_{K^{\prime}(I)}\left[1+G\left(1+F^{\prime}\right)^{-1}\right]\right| \\
& -\left|\prod_{J(I)}\left(1+F^{\prime}\right)-\prod_{K^{\prime}(I)}\left(1+F^{\prime}\right)\right|\left|\prod_{K^{\prime}(I)}\left[1+G\left(1+F^{\prime}\right)^{-1}\right]\right| . \\
> & \beta\left|\prod_{J(I)}\left[1+G\left(1+F^{\prime}\right)^{-1}\right]-\prod_{K^{\prime}(I)}\left[1+G\left(1+F^{\prime}\right)^{-1}\right]\right|-\left[\beta \varepsilon(2 B)^{-1}\right] B,
\end{aligned}
$$

and hence,

$$
\varepsilon>\left|\prod_{J(I)}\left[1+G\left(1+F^{\prime}\right)^{-1}\right]-\prod_{K^{\prime}(I)}\left[1+G\left(1+F^{\prime}\right)^{-1}\right]\right| .
$$

Therefore, the desired product integral exists.

Now, since ${ }_{x} \Pi^{y}\left[1+G\left(1+F^{\prime}\right)^{-1}\right]$ exists for $a \leqq x<y \leqq b$ and $G\left(1+F^{\prime}\right)^{-1}$ is in $O B^{\circ}$ on $[a, b]$, it follows from Lemma 1.10 that $G\left(1+F^{\prime}\right)^{-1}$ is in $O M^{\circ}$ on $[a, b]$. Hence, since $1+F^{\prime}$ is in $O L^{\circ}$, Lemma 1.11 implies that $G \in O M^{\circ}$ on $[a, b]$.

ProOF $(2,3 \rightarrow 1)$. This result is stated as Lemma 1.4 and is proved in a previous paper by the author [5, Theorem 2].

ProOF $(1,3 \rightarrow 2)$. It follows from Lemma 1.1 that $F+G \in O P^{\circ}$ on $[a, b]$. Further, $F+G \in S_{1} \cap S_{2}$ on $[a, b]$, and $-G \in O B^{\circ}$ and $O M^{\circ}$ on $[a, b]$. Therefore, Lemma 1.4 implies that $F \equiv F+G-G$ is in $O M^{\circ}$ on $[a, b]$. 


\section{BIBLIOGRAPHY}

1. W. D. L. Appling, Interval functions and real Hilbert spaces, Rend. Circ. Mat. Palermo (2) 11 (1962), 154-156. MR 27 \#4040.

2. B. W. Helton, Integral equations and product integrals, Pacific J. Math. 16 (1966), 297-322. MR 32 \#6167.

3. - A product integral representation for a Gronwall inequality, Proc. Amer. Math. Soc. 23 (1969), 493-500. MR 40 \#1562.

4. J. C. Helton, Product integrals, bounds and inverses, Texas J. Sci. (to appear).

5. - Bounds for products of interval functions, Pacific J. Math. (to appear).

6. A. Kolmogoroff, Untersuchungen über den Integralbegriff, Math. Ann. 103 (1930), 654-696.

7. J. S. MacNerney, Integral equations and semigroups, Illinois J. Math. 7 (1963), 148173. MR 26 \#1726.

Department of Mathematics, Arizona State University, Tempe, Arizona 85281 